\title{
Integrating Gender-based Violence Screening and Support Into the Research Clinic Setting: Experiences From an HIV Prevention Open-label Extension Trial in Sub-saharan Africa
}

\section{Morgan Garcia ( $\nabla$ mgarcia@fhi360.org )}

Global Health Population and Nutrition, FHI 360 https://orcid.org/0000-0002-7647-6764

\section{Sarah T Roberts}

Research Triangle Institute: RTI International

Ashley J Mayo

Global Health Population and Nutrition, FHI 360

Rachel Scheckter

Global Health Population and Nutrition, FHI 360

\section{Leila E Mansoor}

CAPRISA: Centre for the Aids Programme of Research in South Africa

Thesla Palanee-Phillips

Wits Reproductive Health and HIV Institute

Krishnaveni Reddy

Wits Reproductive Health and HIV Institute

\section{Yuthika Naidoo}

Wits Reproductive Health and HIV Institute

\section{Carolyne Agwau Akello}

Makerere University

\section{Zakir Gaffoor}

SAMRC: South African Medical Research Council

\section{Samantha Siva}

SAMRC: South African Medical Research Council

Chenai Rushwaya

University of Zimbabwe

Kudzai Hlahla

University of Zimbabwe

Jane Jambaya

University of Zimbabwe

\section{Rujeko Makoni}

University of Zimbabwe 


\section{Evans Kachale}

Johns Hopkins University Bloomberg School of Public Health

Margret Ndovie

Tidziwe Centre: University of North Carolina Project

Jabulisile Zuma

Desmond Tutu HIV Foundation

Elizabeth T Montgomery

Research Triangle Institute: RTI International

\section{Research Article}

\section{Keywords:}

Posted Date: March 2nd, 2022

DOI: https://doi.org/10.21203/rs.3.rs-1184001/v1

License: (c) (i) This work is licensed under a Creative Commons Attribution 4.0 International License.

Read Full License 
Integrating gender-based violence screening and support into the research clinic setting: experiences from an HIV prevention open-label extension trial in sub-Saharan Africa 


\section{INTRODUCTION}

2 Research into antiretroviral-based HIV pre-exposure prophylaxis (PrEP) methods has expanded

3 prevention possibilities, introducing a variety of user-initiated methods - and efforts to support effective

4 use of these methods - that are key to reducing HIV incidence among women, who are disproportionately

5 impacted (1). In March 2021, the updated World Health Organization (WHO) guidelines included one of

6 these methods, the monthly dapivirine vaginal ring ("the ring"), as an additional option for cisgender

7 women, laying the foundation for the introduction of the world's first long-acting HIV prevention product

8 (2). Other methods, including injectable cabotegravir (CAB-LA), as well as multipurpose prevention

9 technologies that prevent both pregnancy and HIV or sexually transmitted infections, are also in various

10 stages of development and regulatory review (3-5). There is also a growing body of evidence indicating

11 that gender-based violence (GBV), especially intimate partner violence (IPV), significantly impacts

12 women's willingness and ability to successfully utilize $\operatorname{PrEP}(6,7)$. Socio-economic and structural factors

13 contribute to HIV and GBV incidence, and recent meta-analyses estimate that GBV prevalence among

14 women in sub-Saharan Africa (SSA) is approximately $44.4 \%(8-12)$.

15

16 Due to the syndemic relationship between HIV and GBV, multiple major donors have recently recognized

17 that expanded and improved GBV response and prevention is a key component of comprehensive HIV services (13). Similarly, improvement of these services at research sites where HIV prevention methods are under investigation offers the opportunity to provide more comprehensive care to participants, and potentially to improve adherence to novel PrEP methods. However, limited guidance exists for HIV

21 service providers and research sites seeking to integrate GBV response into their practices.

22

23 Multi-disciplinary groups, such as sexual assault response teams (SART), have long been used to engage

24 disparate organizations in the creation of standard collaborative processes that enhance the quality, 25 accessibility, and predictability of services for violence survivors $(14,15)$. In addition, programming and 
research efforts related to violence response can present a risk of vicarious trauma to researchers and

27 implementors, particularly for those who have also experienced violence $(16,17)$.

In MTN-025/HOPE, an open-label extension trial of the ring among women in SSA, it was thought that open conversation and disclosure fostered by study-specific counseling, coupled with high rates of GBV within communities where the study took place and routine questions examining experiences of violence, would increase the likelihood of GBV disclosure during study participation. To better meet the needs of study participants and staff, efforts were made to improve site teams' GBV response capacity by incorporating lessons learned from SART practice and vicarious trauma prevention into existing site structures through the creation and implementation of a template standard operating procedure (SOP) for GBV response (18). This paper describes the SOP development and implementation process, as well as outcomes of a process evaluation, to broaden the knowledge base regarding support for GBV survivors.

\section{METHODS}

\section{Study population and design}

41 The HIV Open-label Prevention Extension (HOPE) study (NCT02858037) was a phase 3B trial of the 42 ring for HIV prevention, which enrolled 1456 former participants of the MTN-020/ASPIRE

43 (NCT01617096) phase 3 trial at 14 clinical research sites (CRS) in Malawi, South Africa, Uganda, and

44 Zimbabwe (19). HOPE participants were consented per local institutional review board-approved

45 processes prior to enrollment and followed for approximately 12 months. Participants chose whether to use the ring throughout study participation and were supported to make this choice using a client-centered counseling approach (20). HOPE results reinforced the ring's favorable safety profile and illustrated willingness to use the ring, with more than $73 \%$ of participants choosing the ring throughout follow-up, 
52

53

54

55

56

\section{$57 \quad$ SOP design and implementation}

58 To ensure that site teams were prepared to identify and respond to participants who reported GBV, the 59 study management team conducted two needs assessment surveys - one among site staff and one among

60 site leadership - and developed and implemented a template GBV Response SOP based on identified

61 needs, best practices in multidisciplinary GBV response, and international guidelines regarding provision

62 of services for individuals experiencing $\operatorname{GBV}(14,21)$. In addition, two process assessment surveys were

63

64

65

66

67

\section{Participant GBV evaluation}

At quarterly visits, HOPE participants responded to interviewer-administered behavioral questionnaires exploring experiences of physical violence or sexual assault by current or previous partners, as well as any experience of sexual assault, within the past three months. conducted after SOP implementation, one each with site staff and leadership, to evaluate the SOP implementation process. Thirteen of the 14 HOPE sites implemented the SOP; the last site was participating in a separate study of an integrated PrEP and IPV counseling intervention called the Community Health clinic model for Agency in Relationships and Safer Microbicide Adherence (CHARISMA), following separate study-specific procedures and SOPs $(22,23)$. The CHARISMA site was included in the needs assessment but did not complete the process evaluation.

\section{Needs assessment surveys}

To understand existing resources and needs at HOPE sites, site team members were requested via email to complete two needs assessment surveys in Qualtrics. Surveys were anonymous and contained 10-15 questions, depending on respondent experience and resources to report. The staff survey, completed by counselors, nurses, interviewers, and community educators who directly interacted with participants, addressed: GBV training, referral resources and staff requirements for understanding these resources, confidence in responding to GBV, standardized procedures for GBV response, and feelings of support within their teams. The leadership survey, completed by site investigators of record (IoRs), study 
coordinators, sub-investigators, and CRS leaders, focused on GBV training (including trainers and follow-up requirements), referral partners, standardized GBV response procedures, and mechanisms for receiving participant feedback on referral organizations.

\section{SOP template development}

The HOPE GBV Response SOP template provided a framework for sites to increase basic staff training on the dynamics of GBV and the provision of first-line, trauma-informed care, expand and strengthen referral networks through investigation and relationship development, improve staff support to prevent vicarious trauma by establishing regular debrief sessions and psychological support options, encourage participant sensitization on GBV, and build a shared understanding of staff roles and site approaches to identifying and responding to GBV among participants (24).

\section{Key resources, such as the WHO Clinical and Policy Guidelines for Responding to Intimate Partner} Violence and Sexual Violence Against Women and United Nations Population Fund (UNFPA) MultiSectoral Response to GBV Guide, were cross-referenced with results of the needs assessment surveys to identify key components for the SOP template $(21,25)$. This review led to the inclusion of key definitions, training requirements, and procedures for GBV identification, provision of first-line support, referral, and follow-up. In addition, vicarious trauma prevention trainings, such as those provided by the Headington Institute, were reviewed for tools to facilitate a supportive culture to enable site teams to continue providing first-line and long-term follow-up to participants (26).

\section{SOP adaptation}

Study management supported sites to adapt the SOP template to reflect local resources, site structure, and staff roles. Site teams reviewed and revised lists of referral organizations based on existing needs and template SOP recommendations and conducted outreach when needed. Site leadership engaged local organizations to deliver staff trainings and support participant GBV sensitization via on-site visits and 
104 attendance at participant engagement activities (PEAs), which also provided an opportunity for

105 participants to become familiar with referral organizations. Where local resources were limited, study

106 management supported new connections via regional networks, linking teams to individuals and

107 organizations working to improve human rights in study countries. Study management also helped sites

108 make confidential counseling available to staff, assisting in the identification of support organizations,

109 encouraging investment in psychology sessions, and problem solving so that staff could access existing

110 support within their sites. Multiple sites also engaged professional psychologists to improve processes

111 and resources for staff. Site-specific SOPs adapted from the template were finalized with approval from

112 the management team and study site leadership.

\section{SOP process evaluation surveys}

115 At study end, a second set of anonymous surveys was conducted - one each for staff and leadership -

116 comprising 19-25 questions, depending on referral resources and experience with management team

117 support, to assess SOP adaptation and implementation. These surveys were administered to the same

118 categories of staff members and leaders as the first surveys, but respondents differed due to staff turnover

119 and availability. Because the surveys were anonymous, it is not known how many respondents completed

120 both surveys. Topics addressed included trainings, on-site support for staff, confidence in responding to

$121 \mathrm{GBV}$, and standardized site processes.

\section{Analysis}

124 All four surveys were summarized from Qualtrics data using descriptive statistics and synthesis of

125 qualitative responses to open-ended questions. Comparisons were made between responses on the needs

126 and process evaluation surveys to capture changes reported by site staff and leadership. Illustrative quotes

127 are presented in the Results section. 
HOPE participant responses to behavioral questionnaires were summarized using descriptive statistics of GBV reports during study participation and compared with SOP implementation dates to approximate the number of GBV cases that may have been addressed using the SOP. Site PEA reports were reviewed for mention of referral partner engagement and inclusion of GBV-related topics.

\section{RESULTS}

Needs assessment surveys

A total of 34 site staff (1-5 per site) and 17 site leadership representatives completed the baseline needs assessment.

Of staff, 38\% (13/34) had received some previous training in GBV-related topics as part of their work at the research site. Among 26 staff with prior experience supporting participants reporting violence, 21 (80\%) had reached out to site leadership for support, 13 (50\%) reached out to fellow staff and $6(23 \%)$ contacted outside organizations. Staff were split on standardized responses to GBV: 12 of 26 (46\%) reported a standard site-wide process, an additional 12 of $26(46 \%)$ reported following study-specific processes, and 2 of $26(8 \%)$ reported that there was no standard process for GBV response. Nearly all respondents $(92 \% ; 23 / 25)$ reported that there was at least one organization in their area that offered services to survivors of GBV via written referral $(78 \% ; 18 / 23)$ or walk-in $(48 \% ; 11 / 23)$. Only 35\% $(8 / 23)$ of respondents reported using site staff accompaniment during referrals. When asked what they did well when responding to GBV, most respondents mentioned listening $(36 \% ; 9 / 25)$ or supportive counseling $(28 \% ; 7 / 25)$.

Eight (47\%) leadership respondents indicated that staff received prior training on GBV prevention, support, and response, and 71\% (10/14) indicated that there were no SOPs or other standard resources onsite for responding to participants who disclosed GBV. While nearly all respondents $(93 \% ; 13 / 14)$ indicated that their site provided referrals for GBV services, referrals reportedly occurred yearly (8\%; 
1/13) or less $(92 \% ; 12 / 13)$. Of sites with known referral organizations, 54\% (7/13) collected follow-up

156 information on participant contacts with at least some of these organizations. Like staff, site leadership

157 identified counseling as a strength in responding to GBV (58\%; 7/12). Although site leadership indicated

158 few referrals, $58 \%$ (7/12) also cited access to referral options as a strength.

160 Finally, respondents were asked what was needed to improve GBV response at their site. Responses to

161 this open-ended question are outlined thematically in Table 1.

163 Table 1: Site-reported needs for improving GBV response

\begin{tabular}{|l|c|c|c|}
\hline Response category & Site Leadership & Tite Staff (24) & Total \\
\hline & (12) (N) & $\%(\mathrm{~N})$ \\
\hline GBV Training & $33 \%(4)$ & $54 \%(13)$ & $47 \%(17)$ \\
\hline Improved referral networks & $17 \%(2)$ & $21 \%(5)$ & $19 \%(7)$ \\
\hline Further counseling for participants & $33 \%(4)$ & $4 \%(1)$ & $6 \%(2)$ \\
\hline Life skills training for participants & 0 & $8 \%(2)$ & $6 \%(2)$ \\
\hline Staff debriefing & & & $65(2)$ \\
\hline GBV Response SOP & 0 & $8 \%(2)$ & $6 \%(2)$ \\
\hline Material support for participants & $17 \%(2)$ & 0 & $6 \%(2)$ \\
\hline Medical treatment for participants & $17 \%(2)$ & & \\
\hline Other & & & \\
\hline
\end{tabular}


Per standard study practice, all staff cadres were trained on their site-specific SOP. Sites then employed

167 multiple strategies to ensure sufficient GBV training as required by the SOP, including inviting

168 community advisory board members, consultants, and external service organizations to provide trainings,

169 or utilizing online trainings provided by groups such as MOSAIC Training, Service, and Healing Centre

170 and the United Nations High Commissioner for Refugees (UNHCR). Trainings were recommended for all

171 cadres, from outreach workers to clinicians, to foster a supportive research environment. Per SOP

172 recommendations, some sites also initiated multi-disciplinary GBV response teams including staff cadres

173 who received GBV training. Training organizations were added to site referral directories, with staff

174 providing accompanied referrals when accepted by participants.

175

176 In addition, study management provided two mandatory vicarious trauma trainings for the full protocol

177 team. Of seven sites not already offering no-cost professional counseling to staff responding to GBV, five

178 began doing so as a result of SOP implementation. Sites also shared experiences with providing GBV

179 support, including lessons learned from referral provision and follow-up, and preventing vicarious

180 trauma, via presentations during regular multisite HOPE team meetings. Debriefing and problem solving

181 regarding individual and systemic challenges were also provided directly to counselors and site leadership

182 by study management during site assessment visits.

HOPE participant experience

185 Fifty-eight instances of GBV were reported by 52 HOPE participants after SOP implementation,

186 constituting $3.8 \%$ of the 1353 participants with any follow-up visits. The number of cases per site ranged

187 from 1 to 19.

189 Process evaluation surveys

190 Anonymous process evaluation surveys were completed by 17 site leaders and 32 staff after SOP

191 implementation. 
Among staff, 90\% (29/32) reported receiving training specific to GBV, and all site leadership (17/17)

194 reported that trainings had been conducted as part of SOP implementation. Among those responding, site 195 staff reported improved confidence with GBV response $(69 \% ; 18 / 26)$ and increased vicarious trauma 196 support mechanisms available on-site $(61 \% ; 17 / 28)$, including professional counselors and psychologists 197 from outside organizations.

199 "I am now spontaneous in my responses as I am sure of the information I am providing," mentioned one 200 counselor, continuing that "due to debriefing, I am able to give off my best at every intimate partner 201 violence counseling session." Improved support for staff was frequently mentioned, with counselors 202 reporting that "I feel more supported by leadership and do not have grey areas," and "debriefing with other counselors always helps with lifting the burden of being the carrier." Site leadership also mentioned

204 the importance of a supportive atmosphere, stating "the environment has to be welcoming from the onset... participants need to be clearly aware of the assistance they might get from the study team."

Post SOP implementation, 10 of 17 leadership respondents were aware of an instance of GBV at their site, with $60 \%(\mathrm{n}=6)$ reporting that their team's response skills had improved. One IoR cited the coordinated response as a benefit to participants: "IPV was mainly being addressed by clinicians and counselors - now the multi-disciplinary site team has a chance to participate in the management of IPV.”

211 Leadership also mentioned increased knowledge and vicarious trauma prevention resources as benefits to 212 their teams. In addition, most site leadership (89\%; 17/19) mentioned the GBV Response SOP as the 213 primary resource used when responding to GBV at the site. Among the 84\% (16/19) of site leadership 214 respondents who provided feedback on the usefulness of SOP implementation, all reported that the 215 process was helpful for their teams, with one study coordinator noting that the SOP "makes it easier for 216 the site staff to identify the victims and assist in helping them on time," and a CRS leader reporting that 217 this "has guided our management of IPV cases and ensured that we reviewed our referral network to 
ensure adequate referral was in place.” This CRS leader also appreciated the opportunity to conduct a

219 "critical examination of the site preparedness to manage cases," and the attention paid to preventing and

220 addressing vicarious trauma.

222 While 27\% (8/29) of staff respondents reported an increase in known resources to support participants

223 experiencing violence, only $13 \%$ (2/14) of site leadership respondents reported improved referral

224 networks because of the SOP. Noted improvements included smoother referrals and inclusion of

225 additional referral organizations, with site staff mentioning the addition of transport to referral

226 organizations, leadership follow-up on referral outcomes, and provision of referral service pamphlets.

227 Both leadership and staff expressed that a lack of adequate referral organizations - such as those

228 providing financial, legal, or housing support - remained a challenge when assisting participants. Site

229 leadership also highlighted the importance of changing societal norms about GBV to improve its

230 identification and response, with one sub-investigator noting, "women will only become more open about

231 reporting when all degrees of IPV become socially/culturally unacceptable.”

233 Site leadership made multiple recommendations for SOP implementation in future trials across all study

234 populations, including integration of GBV-related topics into all participant engagement activities and

235 monitoring and documentation of all instances of GBV and resulting follow-up.

237 Although the SOP encouraged sites to invite organizations to PEAs, only two sites reported that a GBV

238 service organization attended an event - both sites were in Harare and engaged the same organization.

239 Overall, four sites, two each in Durban and Harare, reported carrying out discussions related to GBV with

240 participants during these events - two as a result of SOP implementation, with three of these sites

241 addressing the topic of GBV multiple times. One counselor emphasized the importance of this

242 collaboration: "the participants have managed to acquire more knowledge on how to take care of 
themselves as we now interact more with the support system since we invite them to address participants

244 [in] retention meetings.”

\section{DISCUSSION}

247 Our experience in HOPE illustrates that the use of tailored SOPs to identify and respond to GBV is a 248 feasible strategy to build standardized GBV services within research sites and potentially within other 249 clinics providing HIV prevention services in a syndemic setting. SOP development can be informed by

250 the use of survey tools and accepted best practices to create a tailored, systematic approach through which

251 services for GBV survivors can be improved, and support systems and clinic atmospheres around GBV 252 can be strengthened to benefit both staff and the populations they serve (27).

HOPE teams were able to utilize their SOPs and reported increased training, improved confidence, and more on-site support to prevent and address vicarious trauma. Leadership at research sites also reported improved response skills among their teams. Enhanced atmospheres of support for participants and staff

257 were noted by site team members when asked to reflect on the SOP process.

The combination of needs and process evaluation surveys also allowed the HOPE team to identify ongoing gaps, such as limited time for providing counseling and follow-up to participants. Leadership reports of insufficient referral networks in the process evaluation, and limited engagement of referral organizations at PEA events, also highlight the importance of adequate community-based referral networks and ongoing efforts to address societal norms that lead to GBV. While clinic teams can be equipped to provide first-line support and offer a comprehensive network of warm referrals, client access to crucial services such as emergency housing and financial assistance, legal and protection services, and ongoing counseling is limited by what is available nearby (28). Efforts to improve local referral networks

267 have had success when referral organizations received advance training and iterative processes for maintaining referral relationships were implemented, however this was outside the scope of the HOPE 
study (29). Furthermore, site services do not improve primary prevention of GBV, and these services may

270 be hampered when GBV is normalized within a community, regardless of efforts to sensitize participants

271 (30). Our experience indicates that future efforts to improve GBV response would benefit from funding

272 dedicated to professional counseling for site staff and collaborations with primary prevention

273 organizations.

275 Our assessment of the SOP process aimed to determine what impacts this process had on site GBV 276 response. However, recall bias and social desirability bias due to the timing of the process evaluation and 277 respondent knowledge that study management would have access to survey results may have impacted 278 findings. In addition, because the SOP development and implementation process was carried out to 279 improve participant services rather than as part of the trial, participants were not randomized to SOP 280 implementation or standard of care, and no data were collected regarding the SOP and its use or impact 281 on participant wellbeing; indicators of improved counseling abilities and increasingly supportive site 282 atmospheres are based on site team report. Finally, there were few identified instances of GBV during

283 HOPE, limiting our ability to explore how GBV response guided by SOP implementation related to HIV 284 prevention and adherence to study product.

Our experience also suggests that supportive research staff and clinic environments for GBV survivors 287 can be fostered as a complement to biomedical HIV prevention research services, even if extensive 288 resources are not available. Although more research is needed, studies suggest that delivering survivor289 centered support to users of biomedical HIV prevention products may support them to continue using 290 PrEP as long as they feel it is necessary, rather than discontinuing use or struggling with consistent use 291 due to partner objection or trauma related to experiences of violence $(6,7,31)$. 
294 The HOPE GBV Response SOP Template (Supplemental File 1) implementation experience contributes 295 to ongoing efforts to expand GBV identification and support in research and service provision related to 296 PrEP (24). The process and experiences described here informed the development of the United States 297 Agency for International Development (USAID)-supported CHARISMA-CHOICE SOP and Job Aid for 298 Addressing Partner Relationships and IPV in PrEP Services (Supplemental File 2), now publicly available 299 (32). More research is needed to understand the client experience with clinic teams utilizing a GBV 300 response SOP and to further understand what works to support staff providing these services in resource301 limited settings. Further investigation into best practices of GBV response teams may reveal additional 302 tools that translate well to HIV prevention settings. We recommend that the approach described here be 303 considered by PrEP implementers and researchers as an efficient and acceptable way to improve GBV 304 identification and response. 


\section{ACKNOWLEDGMENTS}

The authors would like to thank the MTN-025/HOPE study participants and all site team members for their contributions to the important work of HIV prevention and support for individuals experiencing violence. We would also like to MTN-025/HOPE Study Team lead by Jared Baeten (protocol chair), Thesla Palanee-Phillips (protocol co-chair), Nyaradzo Mgodi (protocol co-chair), Elizabeth Brown (protocol statistician), Ashley Mayo (FHI 360), and Lydia Soto-Torres (DAIDS medical officer). We additionally thank site leaders including: Malawi, Blantyre site (Johns Hopkins University, Queen Elizabeth Hospital): Bonus Makanani; Malawi, Lilongwe site (University of North Carolina, Chapel Hill): Lameck Chinula; South Africa, Cape Town site (University of Cape Town): Gonasagrie Nair; South Africa, Durban - Botha's Hill, Chatsworth, Isipingo, Tongaat, Verulam sites (South African Medical Research Council): Gita Ramjee, Logashvari Naidoo, Dishiki Kalonji, Samantha Siva, Nishanta Singh; South Africa, Durban, eThekwini site (Center for the AIDS Programme for Research in South Africa): Leila Mansoor; South Africa, Johannesburg site (Wits RHI): Thesla Palanee-Phillips ; Uganda, Kampala site (John Hopkins University, Makerere University): Brenda Gati Mirembe; Zimbabwe, Chitungwiza and Harare-Zengeza, Seke South and Spilhaus sites (University of Zimbabwe Clinical Trials Research Centre (UZ-CTRC): Nyaradzo Mgodi, Portia Hunidzarira, and Felix Mhlanga. Data management was provided by The Statistical Center for HIV/AIDS Research \& Prevention (Fred Hutchinson Cancer Research Center, Seattle, WA). For qualitative data, management was provided by the Women's Global Health Imperative Program (RTI International, San Francisco, CA). The vaginal rings used in this study were developed and supplied by the International Partnership for Microbicides (IPM). The content is solely the responsibility of the authors and does not necessarily represent the official views of the National Institutes of Health.

\section{Additional files}

Additional file 1: MTN-025/HOPE GBV Response SOP Template

Word document distributed to sites for adaptation and implementation during the MTN-025/HOPE study. 
333 Additional file 2: CHARISMA-CHOICE SOP for Addressing Partner Relationships and IPV in PrEP

334 Services

335 Word document version of the SOP developed by the CHOICE Collaboration, finalized in October 2020.

336

337 List of abbreviations

338 ASPIRE

339 CAB-LA

340 CHARISMA

341 CRS

342 GBV

343 HIV

344 HOPE

345 IOR

346 IPM

347 IPV

348 PEA

349 PrEP

350 SART

351 SOP

352 SSA

353 USAID

354 UNFPA

355 UNHCR

356 WHO 


\section{References}

358 1. Minnis AM, Browne EN, Boeri M, et al. Young Women's Stated Preferences for Biomedical HIV

359 Prevention: Results of a Discrete Choice Experiment in Kenya and South Africa. J Acquir Immune Defic 360 Syndr. 2019;80(4):394-403.

361 2. WHO. Updated recommendations on HIV prevention, infant diagnosis, antiretroviral initiation 362 and monitoring. Geneva: World Health Organization; 2021.

363 3. Clement ME, Kofron R, Landovitz RJ. Long-acting injectable cabotegravir for the prevention of 364 HIV infection. Curr Opin HIV AIDS. 2020;15(1):19-26.

365 4. Highleyman L. Islatravir Shows Promise for Once-Monthly Oral PrEP. POZcom [Internet]. July 366 30, 2021. Available from: https://www.poz.com/article/islatravir-shows-promise-oncemonthly-oral-prep.

367 5. Rosenthal M. Vaginal Ring Delivered Sustained Levels of Dapivirine and Levonorgestrel. 368 Infectious Disease Special Edition [Internet]. July 30, 2021. Available from: https://www.idse.net/HIV369 AIDS---STIs/Article/01-21/Vaginal-Ring-Delivered-Sustained-Levels-of-Dapivirine-and-

$370 \quad$ Levonorgestrel/62360.

371 6. Roberts S, Haberer J, Celum C, et al. Intimate Partner Violence and Adherence to HIV Pre372 exposure Prophylaxis (PrEP) in African Women in HIV Serodiscordant Relationships: A Prospective 373 Cohort Study. J Acquir Immune Defic Syndr. 2016;73:1.

374 7. Cabral A, J MB, Ngure K, et al. Intimate Partner Violence and Self-Reported Pre-exposure 375 Prophylaxis Interruptions Among HIV-Negative Partners in HIV Serodiscordant Couples in Kenya and 376 Uganda. J Acquir Immune Defic Syndr. 2018;77(2):154-9.

377 8. Anderson JC, Campbell JC, Farley JE. Interventions to address HIV and intimate partner violence 378 in Sub-Saharan Africa: a review of the literature. J Assoc Nurses AIDS Care. 2013;24(4):383-90.

379 9. Kacanek D, Bostrom A, Montgomery ET, et al. Intimate Partner Violence and Condom and 380 Diaphragm Nonadherence Among Women in an HIV Prevention Trial in Southern Africa. J Acquir 381 Immune Defic Syndr. 2013;64(4). 
382 10. Maman S, Campbell J, Sweat MD, Gielen AC. The intersections of HIV and violence: directions

383 for future research and interventions. Soc Sci Med. 2000;50(4):459-78.

384 11. Ramjee G, Daniels B. Women and HIV in Sub-Saharan Africa. AIDS Res Ther. 2013;10(1):30.

385 12. Muluneh MD, Stulz V, Francis L, Agho K. Gender Based Violence against Women in Sub-

386 Saharan Africa: A Systematic Review and Meta-Analysis of Cross-Sectional Studies. Int J Environ Res

387 Public Health. 2020;17(3):903.

388 13. OGAC. PEPFAR 2021 Country and Regional Operational Plan (COP/ROP) Guidance for all 389 PEPFAR Countries. USAID; 2021. p. 429-32.

390 14. Horga I. Multi-sectoral response to GBV: An effective and coordinated way to protect and 391 empower GBV victims/survivors. UNFPA EECARO; 2015.

392 15. Zajac JJ. Report on the National Needs Assessment of Sexual Assault Response teams. United 393 States: National Sexual Violence Resource Center March 1, 2006.

394 16. McCann IL, Pearlman LA. Vicarious traumatization: A framework for understanding the 395 psychological effects of working with victims. J Trauma Stress. 1990;3(1):131-49.

396 17. Williamson E, Gregory A, Abrahams H, Aghtaie N, Walker S-J, Hester M. Secondary Trauma: 397 Emotional Safety in Sensitive Research. J Acad Ethics. 2020;18(1):55-70.

398 18. Baeten JM, Palanee-Phillips T, Mgodi NM, et al. Safety, uptake, and use of a dapivirine vaginal 399 ring for HIV-1 prevention in African women (HOPE): an open-label, extension study. Lancet HIV. $400 \quad 2021 ; 8(2): e 87-e 95$.

401 19. Baeten JM, Palanee-Phillips T, Brown ER, et al. Use of a Vaginal Ring Containing Dapivirine for 402 HIV-1 Prevention in Women. N Engl J Med. 2016;375(22):2121-32.

403 20. Balán IC, Giguere R, Lentz C, et al. Client-Centered Adherence Counseling with Adherence 404 Measurement Feedback to Support Use of the Dapivirine Ring in MTN-025 (The HOPE Study). AIDS 405 and Behavior. 2021;25(2):447-58.

406 21. WHO. Responding to intimate partner violence and sexual violence against women: WHO 407 clinical and policy guidelines. Italy: WHO; 2013. 
408 22. Montgomery ET, Roberts ST, Reddy K, et al. Integration of a Relationship-focused Counseling

409 Intervention with Delivery of the Dapivirine Ring for HIV Prevention to Women in Johannesburg:

410 Results of the CHARISMA Pilot Study. AIDS Behav. 2021.

411 23. Wilson EK, Wagner LD, Palanee-Phillips T, et al. Acceptability and feasibility of the

412 CHARISMA counseling intervention to support women's use of pre-exposure prophylaxis: results of a

413 pilot study. BMC Women's Health. 2021;21(1):126.

414 24. MTN. HOPE GBV Response SOP Template. Durham, NC, USA: Microbicide Trials Network; 4152017.

416 25. EEIRH. Multi-sectoral response to GBV: An effective and coordinated way to protect and 417 empower GBV victims/survivors. In: Horga I, editor. Romania: UNFPA; 2015.

418 26. Iqbal M, Lavy T, Evces MR. What Resources Are Available on Vicarious Trauma? Vic Trauma 419 Disaster Mental Health. 1st ed: Routledge; 2015. p. 8.

420 27. O'Campo P, Kirst M, Tsamis C, Chambers C, Ahmad F. Implementing successful intimate 421 partner violence screening programs in health care settings: Evidence generated from a realist-informed 422 systematic review. Soc Sci Med. 2011;72(6):855-66.

423 28. Odero M, Hatcher AM, Bryant C, et al. Responses to and resources for intimate partner violence: 424 qualitative findings from women, men, and service providers in rural Kenya. J Interpers Violence. $425 \quad 2014 ; 29(5): 783-805$.

426 29. Hatcher A, Turan J, Bukusi E, et al. Linking IPV Screening to Services and Care through Referral 427 Networks2014.

428 30. Beydoun HA, Beydoun MA. Invited commentary: disclosure of gender-based violence in 429 developing countries. Am J Epidemiol. 2014;179(5):613-8.

430 31. Wagman JA, King EJ, Namatovu F, et al. Combined Intimate Partner Violence and HIV/AIDS 431 Prevention in Rural Uganda: Design of the SHARE Intervention Strategy. Health Care Women In. $432 \quad 2016 ; 37: 25$. 
433 32. FHI 360, RTI International. Standard Operating Procedure for Addressing Partner Relationships 434 and Intimate Partner Violence in Pre-Exposure Prophylaxis (PrEP) Services. October 2020 ed. Durham, 435 NC, USA: FHI 360; 2020. 


\section{Supplementary Files}

This is a list of supplementary files associated with this preprint. Click to download.

- MTNTemplateSOPforlPV.SA.pdf

- CHARISMACHOICESOPRelationshipsIPVPrEPOctober20204.pdf 